\title{
ON THE EQUIDISTRIBUTION OF SUMS OF INDEPENDENT RANDOM VARIABLES
}

\section{HERBERT ROBBINS ${ }^{1}$}

1. The main theorem. Let $X_{1}, X_{2}, \cdots$ be a sequence of independent, real-valued random variables with a common distribution function $F(x)=\operatorname{Pr}\left[X_{n} \leqq x\right]$, and let $S_{n}=X_{1}+\cdots+X_{n}$. We are going to show in an elementary manner that the sequence $\left\{S_{n}\right\}$ is "equidistributed" on the line $-\infty<x<\infty$ with respect to a certain class $\mathfrak{H C}$ of functions $h(x)$, in the sense that for any $h(x)$ in $\mathfrak{H C}$,

$$
\lim _{n \rightarrow \infty} \frac{1}{n} \sum_{j=1}^{n} h\left(S_{j}\right)=M(h) \quad \text { with probability 1, }
$$

where the constant $M(h)$ is the mean value of $h(x)$ as defined in the theory of almost periodic functions.

Let $\phi(t)=\int e^{i t x} d F(x)$ denote the characteristic function of the $X$ 's. Concerning the roots of the equation $\phi(t)=1$ one of the three following cases must hold.

Case 1 ("General" CASE). $\phi(t)=1$ if and only if $t=0$.

CASE 2 ("LATtice" CASE). $\phi(t)$ is not identically equal to 1 but there exists a value $t_{0} \neq 0$ such that $\phi\left(t_{0}\right)=1$. All the mass of $F(x)$ is then concentrated at one or more of the points $x=2 k \pi / t_{0} \quad(k=0$, $\pm 1, \cdots)$. It can be shown that there exists a largest positive number $\beta, 0<\beta<\infty$, such that all the mass of $F(x)$ is concentrated at one or more of the points $x=k \beta$, and the number $\beta$ has the property that $\phi(t)=1$ if and only if $t=2 k \pi / \beta(k=0, \pm 1, \cdots)$.

CASE 3 (TRIvial CASE). $\phi(t)=1$ for all $t$. All the mass of $F(x)$ is then concentrated at $x=0$ and all the $S_{n}$ are identically 0 . The distinction among these three cases is fundamental in what follows.

Let $F(x)$ be arbitrary but fixed. We define the mean value operator $M(h)$ for certain complex-valued functions $h(x)$ of a real variable $x$, $-\infty<x<\infty$, as follows.

CASE 1.

$$
M(h)=\lim _{T \rightarrow \infty} \frac{1}{2 T} \int_{-T}^{T} h(x) d x
$$

provided this limit exists. In particular, for any real $t$ we have

Presented to the Society, October 25, 1952; received by the editors September 27, 1952 and, in revised form, January 22, 1953.

1 John Simon Guggenheim Memorial Fellow. 


$$
M\left(e^{i t x}\right)= \begin{cases}1 & \text { for } t=0 \\ 0 & \text { otherwise }\end{cases}
$$

\section{CASE 2.}

$$
M(h)=\lim _{N \rightarrow \infty} \frac{1}{2 N+1} \sum_{j=-N}^{N} h(j \beta)
$$

provided this limit exists; in particular,

$$
M\left(e^{i t x}\right)= \begin{cases}1 & \text { for } t=2 k \pi / \beta \\ 0 & \text { otherwise. }\end{cases}
$$

CAse 3.

$$
M(h)=h(0) ; M\left(e^{i t x}\right) \equiv 1 .
$$

We now prove the fundamental

LEMMA. For any $F(x)$ and any real number $t$, the relation

$$
\lim _{n \rightarrow \infty} \frac{1}{n} \sum_{j=1}^{n} e^{i t S_{i}}=M\left(e^{i t x}\right)
$$

holds with probability 1.

Proof. For $t=0$ both sides of (1.1) are equal to 1 . If $t \neq 0$ but $\phi(t)$ $=1$, then we are dealing with Case 2 with $t=2 k \pi / \beta$. The right-hand side of (1.1) is therefore equal to 1 , and since each $X_{n}$, and hence each $S_{n}$, is an integer multiple of $\beta$, the left-hand side of (1.1) is also equal to 1. It remains to consider the case $\phi(t) \neq 1$, in which we must show that with probability 1 the left-hand side of (1.1) has the value 0 . Let

$$
Y_{n}=\frac{1}{n} \sum_{j=1}^{n} e^{i t S_{i}}
$$

Then

$$
\begin{aligned}
\left|Y_{n}\right|^{2}=Y_{n} \bar{Y}_{n} & =\frac{1}{n^{2}} \sum_{j, k=1}^{n} e^{i t\left(S_{k}-S_{j}\right)} \\
& =\frac{1}{n}+\frac{2}{n^{2}} \operatorname{Re}\left[\sum_{j<k} e^{i t\left(X_{j+1}+\cdots+X_{k}\right)}\right],
\end{aligned}
$$

so that

$$
E\left|Y_{n}\right|^{2}=\frac{1}{n}+\frac{2}{n^{2}} \operatorname{Re}\left[\sum_{j<k} \phi^{k-i}(t)\right] .
$$


For any number $b \neq 1$,

$$
\begin{aligned}
\sum_{j<k} b^{k-j} & =\sum_{k=2}^{n} \sum_{j=1}^{k-1} b^{k-j}=b \sum_{k=2}^{n}\left(1+b+b^{2}+\cdots+b^{k-2}\right) \\
& =\frac{b}{1-b} \sum_{k=2}^{n}\left(1-b^{k-1}\right)=\frac{b(n-1)}{1-b}-\left(\frac{b}{1-b}\right)^{2}\left(1-b^{n-1}\right)
\end{aligned}
$$

and hence, setting $b=\phi(t) \neq 1$, we have

$$
\begin{aligned}
E\left|Y_{n}\right|^{2}= & \frac{1}{n}+\frac{2(n-1)}{n^{2}} \operatorname{Re}\left[\frac{b}{1-b}\right] \\
& -\frac{2}{n^{2}} \operatorname{Re}\left[\left(\frac{b}{1-b}\right)^{2}\left(1-b^{n-1}\right)\right] \\
\leqq & \frac{A}{n} \text { for some finite constant } A .
\end{aligned}
$$

It follows that for any $\epsilon>0$,

$$
\operatorname{Pr}\left[\left|Y_{n}\right|>\epsilon\right] \leqq \frac{E\left|Y_{n}\right|^{2}}{\epsilon^{2}} \leqq \frac{A}{n \epsilon^{2}}, \quad \sum_{n=1}^{\infty} \operatorname{Pr}\left[\left|Y_{n^{2}}\right|>\epsilon\right]<\infty,
$$

and hence, by the Borel-Cantelli lemma $[1$, p. 154]

$$
\operatorname{Pr}\left[\lim _{n \rightarrow \infty} Y_{n^{2}}=0\right]=1 \text {. }
$$

But, by a familiar form of argument, given any positive integer $n$ we can choose $m=m(n)$ such that

$$
m^{2} \leqq n<(m+1)^{2} ;
$$

then

$$
\begin{aligned}
& Y_{n}=\frac{1}{n} \sum_{j=1}^{n} e^{i t S_{j}}=\frac{m^{2}}{n} Y_{m^{2}}+\frac{1}{n} \sum_{j=m^{2}+1}^{n} e^{i t S_{j}}, \\
& \left|Y_{n}-\frac{m^{2}}{n} Y_{m^{2}}\right| \leqq \frac{n-m^{2}}{n}<\frac{2 m+1}{m^{2}} \rightarrow 0 \quad \text { as } n \rightarrow \infty
\end{aligned}
$$

Since $m^{2} / n \rightarrow 1$ as $n \rightarrow \infty$ it follows from (1.2) and (1.3) that

$$
\operatorname{Pr}\left[\lim _{n \rightarrow \infty} Y_{n}=0\right]=1 \text {, }
$$

which completes the proof.

Definition. For any fixed distribution function $F(x)$ let $\mathfrak{H C}=\mathfrak{H C}(F)$ 
denote the class of all complex-valued functions $h(x),-\infty<x<\infty$, such that $M(h)$ exists and

$$
\lim _{n \rightarrow \infty} \frac{1}{n} \sum_{j=1}^{n} h\left(S_{j}\right)=M(h) \quad \text { with probability } 1 .
$$

The class $\mathfrak{H C}$ depends on the function $F(x)$ which determines the probability distribution of the sequence $\left\{S_{n}\right\}$ and the definition of $M(h)$. (In the trivial Case 3, $\mathfrak{H}$ consists of all functions $h(x)$.) However, the lemma shows that $\pi C$ certainly contains all the functions $e^{i t x}$. It is easy to verify that $\mathcal{H C}$ is closed under addition, multiplication by complex constants, and conjugation, and that $M$ is a linear and monotone operator on $3 C$. Moreover, we can show without trouble that

(I) The uniform limit $h(x)$ of any sequence $h_{n}(x)$ in $\mathcal{H C}$ is also in $\mathcal{H}$, and $M(h)=\lim _{n \rightarrow \infty} M\left(h_{n}\right)$. More generally,

(II) If $h(x)$ is any real-valued function such that there exist two sequences $h_{n}^{\prime}(x), h_{n}^{\prime \prime}(x)$ in $\Re$ such that

$$
h_{n}^{\prime}(x) \leqq h(x) \leqq h_{n}^{\prime \prime}(x) ; \quad \lim _{n \rightarrow \infty}\left[M\left(h_{n}^{\prime \prime}\right)-M\left(h_{n}^{\prime}\right)\right]=0,
$$

then $h(x)$ is in $\mathcal{H C}$ and $M(h)=\lim _{n \rightarrow \infty} M\left(h_{n}^{\prime}\right)=\lim _{n \rightarrow \infty} M\left(h_{n}^{\prime \prime}\right)$.

Since we know that $\pi$ contains all finite sums of the form

$$
\sum_{k=1}^{r} a_{k} e^{i t_{k} x}
$$

where the $t_{k}$ are arbitrary real numbers, it follows from (I) that $\mathcal{H C}$ contains all almost periodic functions (= uniform limits of sums of the form (1.5)); in particular, $\mathfrak{H C}$ contains all continuous and periodic functions. From (II) it follows that $\mathcal{H C}$ contains certain discontinuous functions. For example, suppose that $h(x)$ is real-valued with period $p$ and is Riemann integrable in the interval $0 \leqq x \leqq p$. Suppose further that Case 1 holds, or that Case 2 holds with $\beta$ not a rational multiple of $p$. Given any integer $n$ we can find two finite sums $h_{n}^{\prime}(x), h_{n}^{\prime \prime}(x)$, each of the form

$$
g(x)=\sum_{k=-r}^{r} a_{k} e^{i 2 k \pi x / p}
$$

and such that

$$
h_{n}^{\prime}(x) \leqq h(x) \leqq h_{n}^{\prime \prime}(x) ; \quad \int_{0}^{p} h_{n}^{\prime \prime}(x) d x-\int_{0}^{p} h_{n}^{\prime}(x) d x<\frac{p}{n} .
$$

For such sums as (1.6) we have in either of the two cases 


$$
M(g)=a_{0}=\frac{1}{p} \int_{0}^{p} g(x) d x,
$$

and hence we can write (1.7) as

$$
h_{n}^{\prime}(x) \leqq h(x) \leqq h_{n}^{\prime \prime}(x) ; \quad M\left(h_{n}^{\prime \prime}\right)-M\left(h_{n}^{\prime}\right)<\frac{1}{n} .
$$

It follows from (II) that $h(x)$ is in $3 C$, and we see by a simple argument that

$$
M(h)=\frac{1}{p} \int_{0}^{p} h(x) d x .
$$

(In Case 1 the relation (1.9) is a trivial consequence of the definition of $M(h)$ and the periodicity of $h(x)$. In the special case of Case 2 in which $p=1$ and all the mass of $F(x)$ is concentrated at the single point $\beta, \beta$ irrational, (1.9) is equivalent to the famous theorem [see 2, pp. 315-316] on the equidistribution mod 1 of the fractional parts of the sequence $n \beta$.)

We summarize our results in

THEOREM 1. The class 30 of functions $h(x)$ such that with probability 1

$$
\lim _{n \rightarrow \infty} \frac{1}{n} \sum_{j=1}^{n} h\left(S_{j}\right)=M(h)
$$

contains all almost periodic functions. Also, if $h(x)$ has period $p$ and is Riemann integrable in the interval $0 \leqq x \leqq p$, then with probability 1

$$
\lim _{n \rightarrow \infty} \frac{1}{n} \sum_{j=1}^{n} h\left(S_{j}\right)=\frac{1}{p} \int_{0}^{p} h(x) d x
$$

provided Case 1 holds, or Case 2 with $\beta$ not a rational multiple of $p$.

As an application of Theorem 1 we shall prove the following

COROLlary. Let $h(x)$ be bounded and such that

$$
\lim _{|x| \rightarrow \infty} h(x)=\alpha
$$

exists, $-\infty<\alpha<\infty$. Then (except in the trivial Case 3) with probability 1 ,

$$
\lim _{n \rightarrow \infty} \frac{1}{n} \sum_{j=1}^{n} h\left(S_{j}\right)=\alpha .
$$

Proof. It will suffice to prove the assertion (1.11) in the special 
case when $\alpha=0$ and $h(x)$ is real and non-negative. Suppose, then, that

$$
0 \leqq h(x) \leqq B<\infty, \quad \lim _{|x| \rightarrow \infty} h(x)=0 .
$$

Given any $\epsilon>0$, choose $T_{0}$ so large that

$$
h(x) \leqq \epsilon \text { for } x \geqq T_{0} .
$$

Let $T$ be any constant larger than $T_{0}$ and define

$$
h^{*}(x)=\left\{\begin{array}{l}
B \text { for }|x|<T_{0}, \\
\epsilon \text { for } T_{0} \leqq|x| \leqq T, \\
\text { periodic with period } 2 T .
\end{array}\right.
$$

We may clearly suppose that $B \geqq \epsilon$; then

$$
h(x) \leqq h^{*}(x) \text { for all } x,
$$

and therefore

$$
\frac{1}{n} \sum_{j=1}^{n} h\left(S_{j}\right) \leqq \frac{1}{n} \sum_{j=1}^{n} h^{*}\left(S_{j}\right) .
$$

The function $h^{*}(x)$ is periodic with period $2 T$ and is Riemann integrable for $-T \leqq x \leqq T$. Moreover, in Case 2 we shall suppose that $T$ is chosen so that $\beta$ is not a rational multiple of $2 T$. Then by Theorem 1 , with probability 1 ,

$$
\begin{aligned}
0 & \leqq \limsup _{n \rightarrow \infty} \frac{1}{n} \sum_{j=1}^{n} h\left(S_{j}\right) \leqq \lim _{n \rightarrow \infty} \frac{1}{n} \sum_{j=1}^{n} h^{*}\left(S_{j}\right)=\frac{1}{2 T} \int_{-T}^{T} h^{*}(x) d x \\
& \leqq \frac{2 B T_{0}+2 \epsilon\left(T-T_{0}\right)}{2 T}<2 \epsilon,
\end{aligned}
$$

provided that

$$
T>\frac{B T_{0}}{\epsilon}
$$

Since $\epsilon$ was arbitrary it follows that with probability 1

$$
\lim _{n \rightarrow \infty} \frac{1}{n} \sum_{j=1}^{n} h\left(S_{j}\right)=0,
$$

which was to be proved.

We remark that any function $h(x)$ satisfying the hypotheses of the Corollary is in $\dddot{F}$ (since $M(h)=\alpha$ and (1.11) holds with probability 1 ) although $h(x)$ need not even be measurable. 
As a special case of the Corollary it follows that for any finite interval $I: a \leqq x \leqq b$,

$$
\lim _{n \rightarrow \infty} \frac{\text { number of terms } S_{1}, \cdots, S_{n} \text { in } I}{n}=0
$$

with probability 1, provided only that the $X^{\prime}$ 's are not identically 0 .

2. Sequences equidistributed modulo $I$. A sequence of constants $\left\{a_{n}\right\}$ lying in a finite interval $I: a \leqq x \leqq b$ is said to be equidistributed in $I$ if for every subinterval $I^{\prime}: a^{\prime} \leqq x \leqq b^{\prime}\left(a \leqq a^{\prime}<b^{\prime} \leqq b\right)$,

$$
\lim _{n \rightarrow \infty} \frac{\text { number of terms } a_{1}, \cdots, a_{n} \text { which lie in } I^{\prime}}{n}=\frac{b^{\prime}-a^{\prime}}{b-a} \text {. }
$$

It follows that for any Riemann integrable function $u(x)$ defined on $I$,

$$
\lim _{n \rightarrow \infty} \frac{1}{n} \sum_{j=1}^{n} u\left(a_{j}\right)=\frac{1}{b-a} \int_{a}^{b} u(x) d x .
$$

For any interval $I: a \leqq x \leqq b$ and any number $x$, not necessarily lying in $I$, let $(x)_{I}$ denote " $x$ modulo $I$ "; that is, let $(x)_{I}$ be the unique number such that $a \leqq(x)_{I}<b$ and such that $x-(x)_{I}$ is an integer multiple of $b-a$. If a sequence of constants $\left\{a_{n}\right\}$ is such that the sequence $\left(a_{n}\right)_{I}$ is equidistributed in $I$, we shall call the sequence $\left\{a_{n}\right\}$ equidistributed modulo $I$.

Let $S_{n}=X_{1}+\cdots+X_{n}$, the $X$ 's being independent random variables with any common distribution function for which Case 1 holds, let $I: a \leqq x \leqq b$ be any finite interval, $I^{\prime}: a^{\prime} \leqq x \leqq b^{\prime}$ any subinterval of $I$, and let $h(x)$ be the function defined for all $x$ as follows:

$$
h(x)=\left\{\begin{array}{l}
1 \text { for } x \text { in } I^{\prime} \\
0 \text { for } x \text { in } I \text { but not in } I^{\prime} \\
\text { periodic with period } b-a \text { for all } x .
\end{array}\right.
$$

It follows from Theorem 1 that with probability 1 ,

(2.1) $\lim _{n \rightarrow \infty} \frac{\text { number of terms }\left(S_{1}\right)_{I}, \cdots,\left(S_{n}\right)_{I} \text { which lie in } I^{\prime}}{n}=\frac{b^{\prime}-a^{\prime}}{b-a}$,

and by denumerability considerations that (2.1) holds with probability 1 simultaneously for all pairs $I, I^{\prime}$ of intervals with rational end points $a \leqq a^{\prime}<b^{\prime} \leqq b$. It then follows by approximating an arbitrary interval from within or from without by a rational interval that (2.1) holds with probability 1 simultaneously for all intervals $I, I^{\prime}$. Thus we have proved 
THEOREM 2. In Case 1, with probability 1 the sequence of random variables $\left\{S_{n}\right\}$ is equidistributed modulo I simultaneously for all finite intervals $I$.

3. Poincaré's roulette theorem revisited. A horizontal circular disk is pivoted at its center to a table. Its circumference is marked off into $2 m$ arcs, alternately red and black, the red arcs of angle $\delta_{1}$ and the black arcs of angle $\delta_{2}$. An arrow fixed to the table points initially to a point 0 on the circumference of the disk. The disk is spun in such a way that the total angle $X$ through which the disk turns is a random variable with a probability density $f(x)$. The probability that the disk comes to rest so that the stationary arrow points to a red point on the circumference is given by the integral

$$
\int h(x) f(x) d x
$$

where

$$
h(x)=\left\{\begin{array}{l}
1 \text { for } 0 \leqq x \leqq \delta_{1}(\text { red arc) } \\
0 \text { for } \delta_{1}<x<\delta_{1}+\delta_{2} \text { (black arc) } \\
\text { periodic with period } \delta_{1}+\delta_{2} .
\end{array}\right.
$$

Poincaré [3, pp. 127-129; see also 4, 5] observed that if $f(x)$ is held fixed while $m \rightarrow \infty$ and $\delta_{i} \rightarrow 0$ in such a way that the ratio $\delta_{1} / \delta_{2}$ remains constant, then the integral (3.1) tends to the limit $\delta_{1} /\left(\delta_{1}+\delta_{2}\right)$. (Poincaré actually assumed that $f(x)$ has a bounded derivative, but this assumption is unnecessary.) Thus, despite the presence of the arbitrary density function $f(x)$, for large $m$ the disk behaves as though it had an approximately uniform distribution of probability around its circumference.

Let $\left\{X_{n}\right\}$ be a sequence of independent random variables with the common probability density $f(x)$, corresponding to a succession of spins of the disk in which the disk is returned after each spin to its initial position with the arrow pointing to 0 . According to the strong law of large numbers, the relation

$$
\begin{gathered}
\lim _{n \rightarrow \infty} \text { [proportion of red stopping points among first } n \text { trials] } \\
=\lim _{n \rightarrow \infty} \frac{1}{n} \sum_{j=1}^{n} h\left(X_{j}\right)=\int h(x) f(x) d x
\end{gathered}
$$

will hold with probability 1 .

Now suppose (as is presumably the case in practice) that the successive spins of angles $X_{1}, X_{2}, \cdots$ are applied without touching the 
disk between spins. After the $n$th spin the disk will have turned through a total angle $S_{n}$, and Theorem 1 shows that, in contrast to (3.3), the relation

$\lim _{n \rightarrow \infty}$ [proportion of red stopping points among first $n$ trials]

$$
=\lim _{n \rightarrow \infty} \frac{1}{n} \sum_{j=1}^{n} h\left(S_{j}\right)=\frac{\delta_{1}}{\delta_{1}+\delta_{2}}
$$

will hold with probability 1. In fact, (3.4) will hold even if the $X$ 's do not have a probability density, provided only that their common distribution function $F(x)$ is such that Case 1 holds or Case 2 holds with $\beta$ not a rational multiple of $2 \pi$. This result is truly in the spirit of Poincaré's "method of arbitrary functions."

4. A general equidistribution problem. For a given distribution function $F(x)$ and pair of functions $h_{1}(x), h_{2}(x)$, when does the limit

$$
\lim _{n \rightarrow \infty} \frac{\sum_{j=1}^{n} h_{1}\left(S_{j}\right)}{\sum_{j=1}^{n} h_{2}\left(S_{j}\right)}
$$

exist (either with probability 1 or in probability, etc.) and have as its value a constant which represents some natural measure of the "relative mean value" of $h_{1}(x)$ compared with $h_{2}(x)$ ? In the special case $h_{2}(x) \equiv 1$ this reduces to the problem of $\$ 1$. There we demanded that the value of $(4.1)$ for $h_{2}(x) \equiv 1$ be equal to the mean value $M\left(h_{1}\right)$ as defined in the three different cases. Similarly, we might now require that the value of (4.1) be equal to

$$
\lim _{T \rightarrow \infty} \frac{\int_{-T}^{T} h_{1}(x) d x}{\int_{-T}^{T} h_{2}(x) d x},
$$

and we might confine ourselves to pairs of functions for which the limit

$$
\lim \frac{\int_{T_{1}}^{T_{2}} h_{1}(x) d x}{\int_{T_{1}}^{T_{2}} h_{2}(x) d x}
$$


exists uniformly as $T_{2}-T_{1} \rightarrow \infty$.

If the individual limits

$$
\lim _{n \rightarrow \infty} \frac{1}{n} \sum_{j=1}^{n} h_{i}\left(S_{j}\right) \quad(i=1,2)
$$

exist and are not both 0 , then (4.1) exists also, so that the results of $\hat{8} 1$ give a partial answer to the present problem. However, the case in which both the limits (4.3) exist and are 0 is often of great interest, and this is not covered by the results of $\$ 1$. We shall be content here with giving three simple examples illustrative of the general problem.

EXAMPLE 1 (Periodic intervals). Let $h_{1}(x)$ be defined by (3.2) and let $h_{2}(x)=1-h_{1}(x)$. In Case 1 , Theorem 1 shows that, with probability 1 , (4.3) exists and equals $\delta_{i}$, so that (4.1) exists and equals the value $\delta_{1} / \delta_{2}$ of (4.2).

EXaMpLE 2 (Infinite intervals). Let $h_{1}(x)=1$ for $x \geqq 0$ and $h_{1}(x)=0$ for $x<0$, while $h_{2}(x)=1-h_{1}(x)$. By a theorem of Erdös and Kac [6], if the $X$ 's have mean 0 and finite second moment, the random variables

$$
Z_{n}=\frac{1}{n} \sum_{j=1}^{n} h_{1}\left(S_{j}\right)
$$

have the continuous limiting distribution function

$$
G(x)=\lim _{n \rightarrow \infty} \operatorname{Pr}\left[Z_{n} \leqq x\right]=\frac{2}{\pi} \arcsin x^{1 / 2}(0 \leqq x \leqq 1) .
$$

Since $\sum_{j=1}^{n} h_{2}\left(S_{j}\right)=n\left(1-Z_{n}\right)$ it follows that neither (4.3) nor (4.1) (since the ratio in (4.1) is equal to $Z_{n} /\left(1-Z_{n}\right)$ ) converges to any constant with probability 1 or in probability.

EXAMPLE 3 (Finite intervals). Let $h_{i}(x)=1$ on some finite interval $\left(a_{i}, b_{i}\right)$ and $h_{i}(x)=0$ elsewhere. In [7] it is shown, for example, that if $F(x)$ is not a lattice distribution function and has mean 0 and finite third absolute moment, then the limit in probability of (4.1) is $\left(b_{1}-a_{1}\right) /\left(b_{2}-a_{2}\right)$, and that under somewhat different conditions on $F(x)$ the limit in probability of (4.1) exists more generally wherever the $h_{i}(x)$ are any two bounded functions in $L^{1}(-\infty, \infty)$, and has as its value (4.2), which is here equal to the ratio of the integrals of the functions $h_{i}(x)$ over $(-\infty, \infty)$, the denominator integral being assumed $\neq 0$. Here the individual limits (4.3) exist but are both 0 . A sharper and more general result was proved later in [14].

5. Extension to compact groups. By using the random ergodic theory of Ulam-von Neumann [8] and Kakutani [9], the results of $\$ 1$ 
can be extended to the case in which the random variables $X_{n}$ have values in any compact topological group.

Let $G$ be a compact (not necessarily commutative) topological group with elements $g$ and Haar measure $\mu, \mu(G)=1$, and let $X_{n}(\omega)$ be a sequence of independent $G$-valued random variables with the same distribution defined on a probability space $\Omega$ of points $\omega$. Put $S_{n}(\omega)=X_{1}(\omega) \cdots X_{n}(\omega)$, where the product is taken in the sense of group multiplication in $G$. From Theorem 1 of [9] it follows that for any continuous function $h(g)$ defined on $G$,

$$
\begin{aligned}
\lim _{n \rightarrow \infty} \frac{1}{n} \sum_{j=1}^{n} h\left(g \cdot X_{1}(\omega) \cdots X_{j}(\omega)\right) & \\
& =\lim _{n \rightarrow \infty} \sum_{j=1}^{n} h\left(g \cdot S_{j}(\omega)\right)=\bar{h}(g, \omega)
\end{aligned}
$$

exists for a.e. $(g, \omega)$ in $G \times \Omega$. The functions

$$
h_{n, \omega}(g)=\frac{1}{n} \sum_{j=1}^{n} h\left(g \cdot S_{j}(\omega)\right)
$$

are equi-uniformly continuous on $G$ for any fixed $\omega$, while from Fubini's theorem it follows that for a.e. $\omega$ the limit (5.1) exists for a.e. $g$. Consequently for a.e. $\omega$ the limit (5.1) exists uniformly for all $g$ in $G$. In particular, setting $g=1$,

$$
\lim _{n \rightarrow \infty} \frac{1}{n} \sum_{j=1}^{n} h\left(S_{j}(\omega)\right)=\bar{h}(\omega)
$$

exists a.e. on $\Omega$.

Let $\phi_{x}(g)=g \cdot x$ denote right multiplication in $G$. The family $\left\{\phi_{X(\omega)} \mid \omega \in \Omega\right\}$ of $\mu$-preserving transformations is said to be ergodic on $G$ if, for every Borel subset $B$ of $G, \mu\left[\phi_{\mathrm{X}(\omega)}(B) \Delta B\right]=0$ for a.e. $\omega$ implies $\mu(B)=0$ or 1 , where $\Delta$ denotes symmetric difference. From Theorem 3 of [9] it follows in the ergodic case that the function $\bar{h}(g, \omega)$ of (5.1) is constant a.e. on $G \times \Omega$, and hence the function $\bar{h}(\omega)$ of (5.2) is constant a.e. on $\Omega$.

Now it is easy to see that the family $\left\{\phi_{X(\omega)} \mid \omega \in \Omega\right\}$ is ergodic on $G$ if $X(\omega)$ has the following property: for any null set $N$ of $\Omega$, the set $\{X(\omega) \mid \omega \in \Omega-N\}$ generates an everywhere dense subgroup of $G$. If $X(\omega)$ does not have this property on $G$, we replace $G$ by the smallest closed subgroup $G_{0}$ with the property that $\operatorname{Pr}\left\{\omega \mid X(\omega) \in G_{0}\right\}=1$; then $X(\omega)$ has the desired property on the group $G_{0}$. Consequently, $\bar{h}(g, \omega)$ is constant a.e. on $G_{0} \times \Omega$ and $\bar{h}(\omega)$ is constant a.e. on $\Omega$. Thus 
$h(\omega)$ is constant a.e. on $\Omega$ even when the family $\left\{\phi_{X(\omega)} \mid \omega \in \Omega\right\}$ is not ergodic on $G$.

Theorem 1 of $\S 1$ for $h(x)$ almost periodic follows by taking as $G$ the van Kampen-Weil $[10,11]$ compactification of the real line $-\infty<x$ $<\infty$ with respect to the function $h(x)$. In this case $h(x)$ corresponds to a continuous function $h(g)$ on the compactification. The case in which $h(g)$ is Riemann integrable on $G$ is treated similarly.

The results of this paper are connected to some extent with work by Lévy [12] and Kawada and Ito [13] who discussed convergence of distribution functions (instead of convergence with probability 1 of sequences of random variables) for the case of the additive group of real numbers modulo 1 and a general compact group respectively.

The author is indebted to Professor S. Kakutani for the results of this section.

6. Equidistribution for general stochastic processes. We can easily extend our equidistribution arguments beyond the case of sums of independent random variables. In fact, let $X(t)$ be any stochastic process with a continuous time parameter $t$, defined for all $t \geqq 0$. For any fixed real $\lambda$ set

$$
Y(t)=\frac{1}{t} \int_{0}^{t} e^{i \lambda X(u)} d u .
$$

Then

$$
E|Y(t)|^{2}=\frac{1}{t^{2}} \int_{0}^{t} \int_{0}^{t} E e^{i \lambda[X(u)-X(v)]} d u d v .
$$

We shall assume that for every $\lambda \neq 0$ there exists a $\delta=\delta(\lambda)>0$ such that as $t \rightarrow \infty$,

$$
E|Y(t)|^{2}=O\left(t^{-\delta}\right) .
$$

Then just as in $\S 1$ we can show that with probability 1 ,

$$
\lim _{n \rightarrow \infty} Y(t)= \begin{cases}1 & \text { for } \lambda=0, \\ 0 & \text { for } \lambda \neq 0 .\end{cases}
$$

It follows that the equidistribution properties which hold in Case 1 for the discrete sequence $\left\{S_{n}\right\}$ continue to hold in integral form for the process $X(t)$.

It is therefore of interest to ask for what processes $X(t)$ the assumption (6.1) is satisfied. We shall consider only the case of a process with stationary increments, in which the distribution of the differ- 
ence $X(u)-X(v)$ depends only on the difference $u-v$. Then for fixed $\lambda$, setting

$$
\phi(t)= \begin{cases}E e^{a[x(t)-x(0)]} & \text { for } t \geqq 0, \\ \Phi(-t) & \text { for } t<0,\end{cases}
$$

we can write

$$
E e^{i \lambda[X(u)-X(v)]}=\phi(u-v),
$$

and hence

$$
\begin{aligned}
E|Y(t)|^{2} & =\frac{1}{t^{2}} \int_{0}^{t} \int_{0}^{t} \phi(u-v) d u d v \\
& =\frac{2}{t^{2}} \operatorname{Re} \int_{0}^{t} \int_{0}^{v} \phi(u-v) d u d v \\
& =\frac{2}{t^{2}} \operatorname{Re} \int_{0}^{t} \int_{0}^{v} \phi(u) d u d v \\
& =\frac{2}{t^{2}} \operatorname{Re} \int_{0}^{t}(t-u) \phi(u) d u \\
& \leqq \frac{2}{t} \int_{0}^{t}|\phi(u)| d u .
\end{aligned}
$$

Hence if for some $\delta>0, \phi(t)=O\left(t^{-b}\right)$ as $t \rightarrow \infty$, then setting $\delta^{\prime}$ $=\min (\delta, 1 / 2)$ we have $\phi(t)=O\left(t^{-\delta^{\prime}}\right)$ and therefore from (6.2),

$$
E|Y(t)|^{2} \leqq \frac{2}{t}+\frac{2 C}{t} \int_{1}^{t} u^{-\delta^{\prime}} d u=O\left(t^{-\delta^{\prime}}\right),
$$

so that (6.1) holds with $\delta$ replaced by $\delta^{\prime}$. This proves

TheOREM 3. If $X(t)$ is a stochastic process with stationary increments, such that for every $\lambda \neq 0$ there exists $a \delta=\delta(\lambda)>0$ for which

$$
E e^{\mathrm{a}[\mathrm{X}(t)-x(0)]}=O\left(t^{-\delta}\right) \text { as } t \rightarrow \infty,
$$

then for any function $h(x)$ which is almost periodic, or periodic and Riemann integrable in its period interval, the relation

$$
\lim _{t \rightarrow \infty} \frac{1}{t} \int_{0}^{t} h(X(u)) d u=M(h)=\lim _{A \rightarrow \infty} \frac{1}{2 A} \int_{-A}^{\Delta} h(x) d x
$$

holds with probability 1.

As a particular case of Theorem 3 we mention the case of Brownian 
movement (Wiener-Lévy) in which the random variable $X(u)-X(v)$ is normally distributed with mean 0 and variance $|u-v|$, so that for $t \geqq 0$

$$
\phi(t)=e^{-\lambda^{2} t / 2}
$$

and

$$
\begin{gathered}
E|Y(t)|^{2}=O\left(t^{-1}\right) . \\
\text { BIBLIOGRAPHY }
\end{gathered}
$$

1. W. Feller, Probability theory and its applications, vol. 1, New York, 1950.

2. H. Weyl, Über die Gleichverteilung von Zahlen mod. Eins, Math. Ann. vol. 77 (1916) pp. 313-352.

3. H. Poincaré, Calcul des probabilités, Paris, 1896.

4. M. Frechet, Methode des fonctions arbitraires, etc., vol. 1 (Part III, Book 2 of Traite $d u$ calcul des probabilites (Borel)), Paris, 1938.

5. B. Hostinsky, Methodes generales du calcul des probabilites (Part LII of Memorial des sciences mathématiques), Paris, 1931.

6. P. Erdös and M. Kac, On the number of positive sums of independent random variables, Bull. Amer. Math. Soc. vol. 53 (1947) pp. 1011-1020.

7. G. Kallianpur and H. Robbins, On the sequence of sums of independent random variables, unpublished.

8. S. Ulam and J. von Neumann, Random ergodic theorems, Bull. Amer. Math. Soc. Abstract 51-9-165.

9. S. Kakutani, Random ergodic theorems and Markoff processes with a stable distribution, Proceedings of the Second Berkeley Symposium on Probability and Statistics, 1950, pp. 247-261.

10. E. R. van Kampen, Almost periodic functions and compact groups, Ann. of Math. (2) vol. 37 (1936) pp. 48-91.

11. A. Weil, Sur les fonctions presque périodiques de v. Neumann, C. R. Acad. Sci. Paris vol. 200 (1935) pp. 38-40.

12. P. Lévy, L'addition des variables aléatoires définies sur une circonférence, Bull. Soc. Math. France vol. 67 (1939) pp. 1-41.

13. Y. Kawada and $\mathrm{K}$. Ito, On the probability distribution on a compact group, Proceedings of the Physico-Mathematical Society of Japan vol. 22 (1940) pp. 977-998.

14. T. Harris and H. Robbins, Ergodic theory of Markov chains with an infinite invariant measure, to appear in Proc. Nat. Acad. Sci. U.S.A.

University of North Carolina and

The Institute For Advanced Study 